\title{
Generalized BRST Symmetry and Gaugeon Formalism for Perturbative Quantum Gravity: Novel Observation
}

\author{
Sudhaker Upadhyay* \\ S. N. Bose National Centre for Basic Sciences, \\ Block JD, Sector III, Salt Lake, Kolkata -700098, India.
}

\begin{abstract}
In this paper the novel features of Yokoyama gaugeon formalism are stressed out for the theory of perturbative quantum gravity in Einstein curved spacetime. The quantum gauge transformations for the theory of perturbative gravity are demonstrated in the framework of gaugeon formalism. These quantum gauge transformations lead to renormalized gauge parameter. Further, we analyse the BRST symmetric gaugeon formalism which embeds more acceptable Kugo-Ojima subsidiary condition. Further, the BRST symmetry is made finite and field-dependent. Remarkably, the Jacobian of path integral under finite and field-dependent BRST symmetry amounts to the exact gaugeon action in the effective theory of perturbative quantum gravity.
\end{abstract}

\section{INTRODUCTION}

The understanding of gravity as a quantum theory is one of the great challenges of physics. In search of full quantum theory of gravity some attempts are made by incorporating some modern concepts, namely, string theory and loop quantum gravity. However, in the mean time, it was realized that these modern concepts also meet some enormous conceptual and technical problems. Although the quantum field theoretic approach was considered originally, it became useless that days because of severe difficulties. Nowadays, it might be worthwhile to reconsider the quantum field theoretical approach when the quantum field theory on curved spacetime is well established. The covariant quantum theory of gravity in curved spacetime in a usual perturbative approach begins with the Einstein-Hilbert theory and expands the full Riemannian metric around a constant background. In this approach, the diffeomorphism invariance of the theory gets translated into a gauge symmetry of the fluctuation [1] and hence, the problem of formulating the corresponding quantum field theory in the Einstein curved spacetime is conceptually no more different than the usual gauge theories. The study of quantum field theory in curved spacetime (particularly in de Sitter spacetime) has significant role in inflationary cosmologies [2 [5]. The recently observed data indicates that the rate of expansion of universe is such that it may approach de Sitter spacetime asymptotically [6]. Further, the gauge invariant perturbative quantum gravity in curved space has founded great attempts to unify gravity with the Maxwell theory [7]. The gauge invariant gravity models have their relevance in certain string theories [8 10].

On the other hand, in the standard quantization of the gauge theories the gauge invariance at the quantum level gets converted into fermionic rigid BRST invariance [11]. Such BRST symmetry plays an important role in the proof of renormalizability and unitarity of the gauge theories [11]. The generalized BRST symmetry, the so-called FFBRST symmetry, has also been studied which has great implications on gauge theories in flat as well as in curved spacetime 12 24]. Recently, the BRST symmetry for the perturbative quantum gravity in the curved spacetime has been analysed [25, 26] and also has been generalized in the FFBRST framework [24].

However, in the quantization of gauge theories, one does not investigate the gauge transformation at the quantum level as the theory does not exhibit quantum gauge freedom. A quantum theory is defined only after fixing a suitable gauge which is parametrize by a gauge parameter. Such gauge condition breaks the local gauge invariance. Hayakawa and Yokoyama have shown that a shift in gauge parameter occurs through renormalization which affects the gauge-fixing condition [27]. Besides this, Yokoyama's

*Electronic address: sudhakerupadhyay@gmail.com; sudhaker@boson.bose.res.in 
gaugeon formalism 28 32 provides the wider framework for quantization of gauge theories. Within the gaugeon formalism, theory admits the quantum gauge transformation under which the shift in the gauge parameter occurs naturally which gets identified with the renormalized gauge parameter [28]. The idea behind gaugeon formalism is to study the quantum gauge freedom by extending the configuration space with the introduction of set of extra fields (so-called gaugeon fields) in the effective Lagrangian density. Since the gaugeon fields are not physical fields as they do not contribute in physical processes, one needs to remove them. Yokoyama first time removed them by putting the extra subsidiary condition of Gupta-Bleuler type which has certain limitations [28]. Further extension of configuration space is made by introducing ghosts corresponding to gaugeon fields [33, 34] and by doing so the Gupta-Bleuler type restriction gets converted into the Kugo-Ojima type restriction [35, 36]. The gaugeon formalism has been studied extensively in many contexts [33, 34, 37 44]. We analyse the gaugeon formalism in the FFBRST framework.

In this paper we consider the diffeomorphism invariant classical gravity theory in the Einstein curved spacetime and discuss the BRST symmetry of the perturbative quantum gravity in covariant gauge. Further, we extend the configuration space by introducing two gaugeon fields which describe the quantum gauge freedom. Such extended Lagrangian density, called the Yokoyama Lagrangian density, possesses quantum gauge transformation under which this remains form invariant. The form invariance of the Yokoyama Lagrangian density leads to a natural shift in gauge parameter which may be identified with the renormalized gauge parameter. As the gaugeon fields do not contribute in physical processes, we put an extra restriction of the Gupta-Bluler type on gaugeon fields. But the Gupta-Bluler type condition is not valid for all cases. Hence we further extend the action by introducing ghost fields for each gaugeon fields to improve the limitations of Gupta-Bleuler condition. This extended action respects both the BRST symmetry and quantum gauge symmetry. Now, we generalize the BRST transformation by making the parameter finite and field-dependent. Under such generalized BRST transformation the functional integral does not remain invariant and therefore we calculate the field-dependent Jacobian for the path integral. We therefore found that under generalized BRST transformation, with a particular choice of the finite field-dependent parameter, the Jacobian of path integral leads to BRST symmetric gaugeon extended action. Therefore, we claim that the extended action within gaugeon formalism can be constructed simply by calculating Jacobian under generalized BRST transformation.

We organize the paper in following manner. In section II, we discuss the perturbative quantum gravity in gaugeon formalism. In Section III, we analyse briefly the mechanism of generalized BRST transformation. The emergence of gaugeon action through Jacobian is calculated explicitly in section IV. In the last section we draw the final remarks.

\section{THE PERTURBATIVE QUANTUM GRAVITY IN GAUGEON FORMALISM}

In this section, we develop the theory of perturbative gravity manifestly in the quantum gauge invariant framework. For this purpose, we analyse the perturbative gravity in the Yokoyama gaugeon formalism which possesses the quantum gauge transformation. Let us begin with the Lagrangian density for the classical gravity

$$
\mathcal{L}_{i n v}=\sqrt{-\tilde{g}}(R-2 \Lambda),
$$

where $\tilde{g}$ is the determinant of the full metric $\tilde{g}_{a b}, R$ is the Ricci scalar curvature and $\Lambda$ is the cosmological constant. The units are adopted here such that $16 \pi G=1$.

Further, we decompose the full metric into its background and perturbation parts as follows

$$
\tilde{g}_{a b}=g_{a b}+h_{a b},
$$

where $g_{a b}$ is the fixed background metric and $h_{a b}$ describes the small perturbations around fixed metric. Here the background metric $g_{a b}$ is assumed to satisfy the equation of motion of 1 , that is, the Einstein equation $R_{a b}=\Lambda g_{a b}$. This small perturbation $h_{a b}$ is considered as a field which has to be quantized. 
Now, we expand the Lagrangian density (1) to second order in $h_{a b}$ and thus obtain the Lagrangian density for the linearized gravity as

$$
\mathcal{L}_{i n v}=\sqrt{-g}\left[\frac{1}{2} \nabla^{b} h^{a c} \nabla_{a} h_{b c}-\frac{1}{4} \nabla_{a} h_{b c} \nabla^{a} h^{b c}+\frac{1}{4}\left(\nabla^{a} h-2 \nabla^{b} h_{b}^{a}\right) \nabla_{a} h+\frac{1}{2} \Lambda\left(h_{a b} h^{a b}-\frac{1}{2} h^{2}\right)\right],
$$

with $h=h_{a}^{a}$. This linearized Lagrangian density possesses the following gauge invariance (up to total divergence)

$$
\delta_{\rho} h_{a b}=\nabla_{a} \rho_{b}+\nabla_{b} \rho_{a},
$$

where $\nabla_{a}$ denotes the background covariant derivative and $\rho_{a}$ is a vector field. Analogous to the usual gauge theory where the Lagrangian remains invariant under gauge transformations, we treat the theory of perturbative gravity as a gauge theory which is invariant under coordinate transformations. Now, according to the standard quantization procedure, the gauge invariance of the effective theory reflects the redundancy in the physical degrees of freedom. These unphysical degrees of freedom lead to constraints in the canonical quantization [11] and divergences in the generating functional under the path integral quantization. To resolve this problem one needs to break the gauge invariance by fixing a gauge. In this case we choose the following covariant gauge

$$
G[h]_{a}=\left(\nabla^{b} h_{a b}-k \nabla_{a} h\right)=0,
$$

where $k \neq 1$ is a gauge parameter. Since, for $k=1$, one of the conjugate momenta corresponding to $h_{a b}$ vanishes, this leads to divergence in the partition function. In this view $k$ is written sometimes in terms of an arbitrary finite constant $\beta$ as $(1+\beta) / \beta$ [47]. Now, the gauge condition (5) is incorporated by adding a covariant gauge-fixing term in the Lagrangian density as follows [47]

$$
\mathcal{L}_{g f}=\sqrt{-g}\left[b^{a}\left(\nabla^{b} h_{a b}-k \nabla_{a} h\right)+\frac{\alpha}{2} b_{a} b^{a}\right],
$$

where $\alpha$ is also a gauge parameter and $b^{a}$ is a Lagrange multiplier field. To compensate the contribution of this gauge-fixing term within the functional integral we add the following Faddeev-Popov ghost term in the resulting action

$$
\begin{aligned}
\mathcal{L}_{g h} & =\sqrt{-g} \bar{c}^{a} \nabla^{b}\left[\nabla_{a} c_{b}+\nabla_{b} c_{a}-2 k g_{a b} \nabla_{c} c^{c}\right], \\
& =\sqrt{-g} \bar{c}^{a} M_{a b} c^{b},
\end{aligned}
$$

where the Faddeev-Popov matrix operator $M_{a b}$ has the following form:

$$
M_{a b}=\nabla_{c}\left[\delta_{b}^{c} \nabla_{a}+g_{a b} \nabla^{c}-2 k \delta_{a}^{c} \nabla_{b}\right] .
$$

Now, the total effective Lagrangian for perturbative quantum gravity in covariant gauge can be given as the sum of gauge invariant, gauge-fixing and ghost terms as follows

$$
\mathcal{L}_{T}=\mathcal{L}_{\text {inv }}+\mathcal{L}_{g f}+\mathcal{L}_{g h}
$$

This effective Lagrangian density is invariant under following nilpotent BRST $(s)$ transformation:

$$
s h_{a b}=\nabla_{a} c_{b}+\nabla_{b} c_{a}, \quad s c^{a}=-c_{b} \nabla^{b} c^{a}, \quad s \bar{c}^{a}=b^{a}, \quad s b^{a}=0 .
$$

Utilizing this BRST symmetry transformation we ensure that the gauge-fixing and ghost terms of the complete Lagrangian density are BRST-exact and can be expressed as

$$
\begin{aligned}
\mathcal{L}_{g} & :=\mathcal{L}_{g f}+\mathcal{L}_{g h}, \\
& =s \Psi,
\end{aligned}
$$

where $\Psi$ is the gauge-fixing fermion for the theory of perturbative quantum gravity with the following expression:

$$
\Psi=\sqrt{-g}\left[\bar{c}^{a}\left(\nabla^{b} h_{a b}-k \nabla_{a} h+\frac{\alpha}{2} b_{a}\right)\right] .
$$

Here we note that any physical quantity does not depend on the choice of gauge-fixing fermion [11]. However, the invariance of perturbative quantum gravity under BRST symmetry plays a crucial role in constructing the physical states of the theory. 


\section{A. Yokoyama Gaugeon formalism}

In this subsection, we review the Yokoyama gaugeon formalism for perturbative quantum gravity in the Einstein curved spacetime as discussed in [37]. For this purpose, we start by constructing the Yokoyama Lagrangian density for perturbative quantum gravity as

$$
\begin{aligned}
\mathcal{L}_{y k} & =\mathcal{L}_{i n v}+\sqrt{-g} b^{a}\left(\nabla^{b} h_{a b}-k \nabla_{a} h\right)+\frac{\varepsilon}{2} \sqrt{-g}\left(y_{\star}^{a}+\lambda b^{a}\right)^{2}+\sqrt{-g} \bar{c}^{a} M_{a b} c^{b} \\
& +\sqrt{-g} \nabla^{b} y_{\star}^{a}\left[\nabla_{a} y_{b}+\nabla_{b} y_{a}-2 k g_{a b} \nabla_{c} y^{c}\right],
\end{aligned}
$$

which is an extended version of total effective Lagrangian density (9) having two extra gaugeon fields $y^{a}$ and $y_{\star}^{a}$ satisfying Bose-Einstein statistics. $\varepsilon(= \pm)$ is a sign factor and the original gauge parameter $\alpha$ (given in (6) ) corresponds to $\varepsilon \lambda^{2}$.

Now, the quantum gauge transformations for the Lagrangian density (13), under which the gauge parameter gets shifted, are demonstrated as

$$
\begin{aligned}
& h_{a b} \rightarrow \hat{h}_{a b}=h_{a b}-\tau\left(\nabla_{a} y_{b}+\nabla_{b} y_{a}\right), \\
& y_{\star}^{a} \rightarrow \hat{y}_{\star}^{a}=y_{\star}^{a}-\tau b^{a}, \\
& y_{a} \rightarrow \hat{y}_{a}=y_{a}, \\
& b^{a} \rightarrow \hat{b}^{a}=b^{a}, \\
& \bar{c}^{a} \rightarrow \hat{\bar{c}}^{a}=\bar{c}^{a}, \\
& c^{a} \rightarrow \hat{c}^{a}=c^{a},
\end{aligned}
$$

where the infinitesimal parameter of transformation $\tau$ is bosonic in nature. Under these transformations the Lagrangian given in (13) is "form invariant", i.e. it transforms as

$$
\mathcal{L}_{y k}(\hat{\phi}, \hat{\lambda})=\mathcal{L}_{y k}(\phi, \lambda)
$$

where $\hat{\phi}$ denotes the quantum gauge transformed collective field $\phi$ and the shifted parameter $\hat{\lambda}$ is defined by

$$
\hat{\lambda}=\lambda+\tau \text {. }
$$

The form invariance implies that the quantum fields $\hat{\phi}$ and $\phi$ satisfy the same equations of motion with gauge parameters $\hat{\lambda}$ and $\lambda$ respectively.

It is easy to check that the Yokoyama Lagrangian density (13) is also invariant under the following nilpotent BRST transformation

$$
\begin{aligned}
s h_{a b} & =\nabla_{a} c_{b}+\nabla_{b} c_{a}, \quad s \bar{c}^{a}=b^{a} \\
s c^{a} & =-c_{b} \nabla^{b} c^{a}, \quad s b^{a}=0, \\
s y^{a} & =0, \quad s y_{\star}^{a}=0 .
\end{aligned}
$$

Excluding the BRST variation of Yokoyama fields, the above symmetry transformations are identical to those in 10) .

To remove the unphysical modes and define physical states, we impose two subsidiary conditions:

$$
\begin{aligned}
& \left.Q_{b} \mid \text { phys }\right\rangle=0, \\
& \left.y_{\star}^{a(+)} \mid \text { phys }\right\rangle=0,
\end{aligned}
$$

where $Q_{b}$ is Noether's conserved charge corresponding to the BRST symmetry (17). However, the second subsidiary condition (of the Gupta-Bleuler type) works only when (i) the background spacetime is flat, 
which means the cosmological constant should be zero and (ii) the field $y_{\star}^{a}$ satisfies the following free field equation:

$$
\nabla_{b} \nabla^{b} y_{\star}^{a}=0,
$$

which can be established by exploiting equations of motion of $y^{a}$. If the free field equation does not hold, the decomposition of field $y_{\star}^{a}$ into the positive and negative frequency parts is no more valid. In Eq. (18), the first Kugo-Ojima type condition is used to remove the unphysical gauge field modes from the total Fock space [46]; however, the second Gupta-Bleuler type condition is used for the unphysical gaugeon modes.

\section{B. BRST symmetric gaugeon formalism}

In this subsection, we discuss the BRST symmetric gaugeon formalism for perturbative quantum gravity. For this purpose, we first extend the effective Yokoyama Lagrangian density by introducing two Faddeev-Popov ghosts $K_{\star}^{a}$ and $K^{a}$ corresponding to the gaugeon fields as follows:

$$
\begin{aligned}
\mathcal{L}_{y k b} & =\mathcal{L}_{i n v}+\sqrt{-g} b^{a}\left(\nabla^{b} h_{a b}-k \nabla_{a} h\right)+\frac{\varepsilon}{2} \sqrt{-g}\left(y_{\star}^{a}+\lambda b^{a}\right)^{2} \\
& +\sqrt{-g} \bar{c}^{a} M_{a b} c^{b}+\sqrt{-g} \nabla^{b} y_{\star}^{a}\left(\nabla_{a} y b+\nabla_{b} y_{a}-2 k g_{a b} \nabla_{c} y^{c}\right) \\
& +\sqrt{-g} K_{\star}^{a}\left(\nabla^{b} \nabla_{a} K_{b}+\nabla_{b} \nabla^{b} K_{a}-2 k g_{a b} \nabla^{b} \nabla_{c} K^{c}\right),
\end{aligned}
$$

This extended Lagrangian density is invariant under the following infinitesimal BRST transformation:

$$
\begin{aligned}
& s h_{a b}=\nabla_{a} c_{b}+\nabla_{b} c_{a}, \\
& s c^{a}=-c_{b} \nabla^{b} c^{a}, \quad s \bar{c}^{a}=b^{a}, \\
& s b^{a}=0, s y^{a}=-K^{a}, s y_{\star}^{a}=0, \\
& s K_{\star}^{a}=y_{\star}^{a}, \quad s K^{a}=0,
\end{aligned}
$$

where gaugeon fields form BRST quartet. It is easy to check the nilpotency (i.e. $s^{2}=0$ ) of the above BRST transformation.

Thereafter, we see that the sum of gauge-fixing and ghost parts of the above Lagrangian density is BRST-exact and therefore can be written in terms of the BRST variation of extended gauge-fixing fermion $\Psi$ as

$$
\mathcal{L}_{y k b}=\mathcal{L}_{i n v}+s \Psi
$$

where the extended gauge-fixing fermion has the following expression

$$
\begin{aligned}
\Psi & =\sqrt{-g}\left[\bar{c}^{a}\left(\nabla^{b} h_{a b}-k \nabla_{a} h\right)+\frac{\varepsilon \lambda}{2} \bar{c}^{a}\left(y_{a \star}+\lambda b_{a}\right)\right. \\
& \left.-K_{\star}^{a}\left(\nabla^{b} \nabla_{a} y_{b}+\nabla_{b} \nabla^{b} y_{a}-2 k g_{a b} \nabla^{b} \nabla_{c} y^{c}\right)+\frac{\varepsilon}{2} K_{\star}^{a}\left(y_{\star a}+\lambda b_{a}\right)\right] .
\end{aligned}
$$

This extended gauge-fixing fermion depends on both graviton and gaugeon fields. However, for vanishing gaugeon fields it identifies with the original gauge-fixing fermion (12).

Now, with the help of Noether's charge $(Q)$ corresponding to the above BRST symmetry, we define the Kugo-Ojima type physical subsidiary condition

$$
Q \mid \text { phys }\rangle=0 .
$$

This single subsidiary condition removes both the unphysical gaugeon and unphysical graviton (gauge) modes from the physical subspace of states. For example, it can be seen from expression (21) that the 
gaugeon fields $y, y_{\star}, K$ and $K_{\star}$ form a BRST quartet which appear only as zero-normed states in the physical subspace [46]. Therefore, the condition (24) works fine and does not have any kind of limitations like the Gupta-Bleuler one.

Further, the extended Lagrangian density given in (20) remains form invariant under following quantum gauge transformations

$$
\begin{aligned}
& h_{a b} \rightarrow \hat{h}_{a b}=h_{a b}-\tau\left(\nabla_{a} y_{b}+\nabla_{b} y_{a}\right), \\
& y_{\star}^{a} \rightarrow \hat{y}_{\star}^{a}=y_{\star}^{a}-\tau b^{a}, \\
& y_{a} \rightarrow \hat{y}_{a}=y_{a}, \\
& b^{a} \rightarrow \hat{b}^{a}=b^{a}, \\
& \bar{c}^{a} \rightarrow \hat{\bar{c}}^{a}=\bar{c}^{a}, \\
& c^{a} \rightarrow \hat{c}^{a}=c^{a}+\tau K^{a}, \\
& K_{\star}^{a} \rightarrow \hat{K}_{\star}^{a}=K_{\star}^{a}-\tau \bar{c}^{a}, \\
& K^{a} \rightarrow \hat{K}^{a}=K^{a}, \\
& \lambda \rightarrow \hat{\lambda}=\lambda+\tau .
\end{aligned}
$$

These transformations commute with the BRST transformation given in (21). Consequently, the BRST charge $Q$ also remains unchanged under the above quantum gauge transformations. Therefore, the physical space of states annihilated by charge remains intact with these transformations. Hence, we conclude that the physical Hilbert space of the theory remains unchanged under these quantum gauge transformations.

\section{THE GENERALIZED BRST TRANSFORMATION}

In this section, we analyse the generalization of usual BRST transformation by making the infinitesimal BRST parameter finite and field-dependent. Such a generalized BRST transformation is known as the finite-field-dependent BRST (FFBRST) transformation [12]. The methodology of FFBRST transformation is as follows. We first define the usual BRST transformation characterized by a Grassmann parameter $\delta \Lambda$, written compactly as

$$
\delta_{b} \phi=\mathcal{R}[\phi] \delta \Lambda,
$$

where $\mathcal{R}[\phi]$ is the Slavnov variation of collective field $\phi$. However, the properties of this transformation do not depend on whether the parameter $\delta \Lambda$ is (i) finite or infinitesimal, (ii) field-dependent or not, as long as it is anticommuting and space-time independent. These observations give us liberty to make the infinitesimal parameter $\delta \Lambda$ finite and field-dependent without affecting its Grassmannian nature. To generalize the BRST transformation (26) we start by making the infinitesimal parameter field-dependent with the introduction of an arbitrary parameter $\kappa(0 \leq \kappa \leq 1)$. We allow the fields, $\phi(x, \kappa)$, to depend on $\kappa$ in such a way that $\phi(x, \kappa=0)=\phi(x)$ and $\phi(x, \kappa=1)=\phi^{\prime}(x)$, the transformed field.

The usual infinitesimal field-dependent BRST transformation thus can be constructed generically as

$$
\frac{d \phi(x, \kappa)}{d \kappa}=\mathcal{R}[\phi(x)] \Theta^{\prime}[\phi(x, \kappa)]
$$

where the $\Theta^{\prime}[\phi(x, \kappa)] d \kappa$ is the infinitesimal but field-dependent parameter. Now, the FFBRST transformation $\left(\delta_{f}\right)$ is constructed by integrating the above transformation from $\kappa=0$ to $\kappa=1$, as follows

$$
\phi^{\prime} \equiv \phi(x, \kappa=1)=\phi(x, \kappa=0)+\mathcal{R}[\phi(x)] \Theta[\phi(x)],
$$

which can further be written as

$$
\delta_{f} \phi=\phi^{\prime}(x)-\phi(x)=\mathcal{R}[\phi(x)] \Theta[\phi(x)],
$$


where

$$
\Theta[\phi(x)]=\int_{0}^{1} d \kappa^{\prime} \Theta^{\prime}\left[\phi\left(x, \kappa^{\prime}\right)\right]
$$

is the finite field-dependent parameter. Following the procedure discussed above, the FFBRST transformations for the perturbative quantum gravity are constructed from the the infinitesimal BRST transformation (21) as

$$
\begin{aligned}
& \delta_{f} h_{a b}=\left(\nabla_{a} c_{b}+\nabla_{b} c_{a}\right) \Theta[\phi(x)], \\
& \delta_{f} c^{a}=-c_{b} \nabla^{b} c^{a} \Theta[\phi(x)], \\
& \delta_{f} \bar{c}^{a}=b^{a} \Theta[\phi(x)], \quad \delta_{f} b^{a}=0, \\
& \delta_{f} y^{a}=-K^{a} \Theta[\phi(x)], \quad \delta_{f} y_{\star}^{a}=0, \\
& \delta_{f} K_{\star}^{a}=y_{\star}^{a} \Theta[\phi(x)], \quad \delta_{f} K^{a}=0,
\end{aligned}
$$

where $\Theta[\phi(x)]$ is an arbitrary finite field-dependent parameter. Such FFBRST transformation with the finite field-dependent parameter is the symmetry of the effective action. However, the path integral measure is not invariant under such transformation leading to field-dependent Jacobian [12].

The Jacobian $J(\kappa)$ of the path integral measure $\mathcal{D} \phi$ in the functional integral for such transformations is then evaluated for the arbitrary finite field-dependent parameter $\Theta[\phi(x)]$ as

$$
\mathcal{D} \phi^{\prime}=J(\kappa) \mathcal{D} \phi(\kappa),
$$

where the Jacobian can be replaced (within the functional integral) as

$$
J(\kappa) \rightarrow \exp \left[i S_{1}[\phi(x, \kappa)]\right],
$$

for some local functional $S_{1}[\phi]$, if and only if the following condition is satisfied [12]

$$
\int \mathcal{D} \phi(x)\left[\frac{1}{J} \frac{d J}{d \kappa}-i \frac{d S_{1}[\phi(x, \kappa)]}{d \kappa}\right] \exp \left[i\left(S_{e f f}+S_{1}\right)\right]=0 .
$$

This condition is very crucial which preserves the consistency of transformed functional integral with original functional integral.

Using the Taylor expansion, the infinitesimal change in $J(\kappa)$ is derived as follows (for explicit derivation see e.g. [12])

$$
\frac{1}{J(\kappa)} \frac{d J(\kappa)}{d \kappa}=-\int d^{4} x\left[ \pm \mathcal{R}[\phi(x)] \frac{\partial \Theta^{\prime}[\phi(x, \kappa)]}{\partial \phi(x, \kappa)}\right],
$$

where + sign is used for bosonic fields and - sign is used for fermionic fields.

Henceforth, we observe that under the FFBRST transformation with the field-dependent parameter $\Theta$, the functional integral transforms as

$$
\int D \phi e^{i \int d^{4} x \mathcal{L}_{T}} \stackrel{F F B R S T}{\longrightarrow} \iint J(\kappa) D \phi e^{i \int d^{4} x \mathcal{L}_{T}} \equiv \int D \phi e^{i \int d^{4} x \mathcal{L}_{T}+i S_{1}[\phi]},
$$

where $\left(\int d^{4} x \mathcal{L}_{T}+S_{1}[\phi]\right)$ is an extended effective action. It means under FFBRST transformation the original effective action of linear gravity $\left(\int d^{4} x \mathcal{L}_{T}\right)$ gets transformed into an extended effective action. However, to produce the extra piece $S_{1}[\phi, \varphi]$ in the effective action having some extra fields $\varphi$ through the Jacobian calculation, we first insert a well-defined path integral measure corresponding to the extra fields (i.e. $\int D \varphi$ ) in the functional integral by hand before performing FFBRST transformation. 


\section{EMERGENCE OF BRST SYMMETRIC YOKOYAMA GRAVITY THEORY}

In this section, we show that for a particular choice of finite field-dependent BRST parameter the Jacobian of path integral measure leads to the gaugeon term (within a functional integral) in the effective theory of the perturbative gravity naturally. For this purpose, our specific choice of finite field-dependent parameter is obtainable from the following infinitesimal field-dependent parameter

$$
\begin{aligned}
\Theta^{\prime}[\phi, \varphi] & =i \gamma \int d^{4} y \sqrt{-g}\left[\frac{\varepsilon \lambda}{2} \bar{c}^{a}\left(y_{a \star}+\lambda b_{a}\right)-K_{\star}^{a}\left(\nabla^{b} \nabla_{a} y_{b}+\nabla_{b} \nabla^{b} y_{a}-2 k g_{a b} \nabla^{b} \nabla_{c} y^{c}\right)\right. \\
& \left.-\frac{\varepsilon}{2} K_{\star}^{a}\left(y_{\star a}+\lambda b_{a}\right)\right],
\end{aligned}
$$

where an arbitrary parameter $\gamma$ is considered to make it more general and $\varphi$ corresponds to the gaugeon fields $\left(y, y_{\star}, K, K_{\star}\right)$ collectively. Furthermore, we calculate the infinitesimal change in Jacobian for this particular choice of field-dependent parameter as follows

$$
\begin{aligned}
\frac{1}{J(\kappa)} \frac{d J(\kappa)}{d \kappa} & =-i \gamma \int d^{4} x \sqrt{-g}\left[-\frac{\varepsilon}{2} \lambda b_{a}\left(y_{\star}^{a}+\lambda b^{a}\right)+y_{\star}^{a}\left(\nabla^{b} \nabla_{a} y_{b}+\nabla_{b} \nabla^{b} y_{a}-2 k g_{a b} \nabla^{b} \nabla_{c} y^{c}\right)\right. \\
& \left.-K_{\star}^{a}\left(\nabla^{b} \nabla_{a} K_{b}+\nabla_{b} \nabla^{b} K_{a}-2 k g_{a b} \nabla^{b} \nabla_{c} K^{c}\right)-\frac{\varepsilon}{2} y_{\star}^{a}\left(y_{\star a}+\lambda b_{a}\right)\right] \\
& =i \gamma \int d^{4} x \sqrt{-g}\left[\frac{\varepsilon}{2}\left(y_{\star}^{a}+\lambda b^{a}\right)^{2}+\nabla^{b} y_{\star}^{a}\left(\nabla_{a} y_{b}+\nabla_{b} y_{a}-2 k g_{a b} \nabla_{c} y^{c}\right)\right. \\
& \left.+K_{\star}^{a}\left(\nabla^{b} \nabla_{a} K_{b}+\nabla_{b} \nabla^{b} K_{a}-2 k g_{a b} \nabla^{b} \nabla_{c} K^{c}\right)\right]
\end{aligned}
$$

where we have utilized the formula (35). Now, we make an ansatz for the local functional $S_{1}$ which appears in the exponential of Jacobian given in (33) as

$$
\begin{aligned}
S_{1}[\phi(x, \kappa), \varphi(x, \kappa), \kappa] & =\int d^{4} x\left[\xi_{1}(\kappa)\left(y_{\star}^{a}+\lambda b^{a}\right)^{2}+\xi_{2}(\kappa) \nabla^{b} y_{\star}^{a}\left(\nabla_{a} y_{b}+\nabla_{b} y_{a}-2 k g_{a b} \nabla_{c} y^{c}\right)\right. \\
& \left.+\xi_{3}(\kappa) K_{\star}^{a}\left(\nabla^{b} \nabla_{a} K_{b}+\nabla_{b} \nabla^{b} K_{a}-2 k g_{a b} \nabla^{b} \nabla_{c} K^{c}\right)\right],
\end{aligned}
$$

where $\xi_{i}, i=1,2,3$ are arbitrary $\kappa$-dependent constants and satisfy initial boundary conditions $\xi_{i}(\kappa=$ $0)=0$. Now, exploiting relation (27), the infinitesimal change in above functional with respect to $\kappa$ is given by

$$
\begin{aligned}
\frac{d S_{1}}{d \kappa} & =\int d^{4} x\left[\frac{d \xi_{1}}{d \kappa}\left(y_{\star}^{a}+\lambda b^{a}\right)^{2}+\frac{d \xi_{2}}{d \kappa} \nabla^{b} y_{\star}^{a}\left(\nabla_{a} y_{b}+\nabla_{b} y_{a}-2 k g_{a b} \nabla_{c} y^{c}\right)\right. \\
& +\frac{d \xi_{3}}{d \kappa} K_{\star}^{a}\left(\nabla^{b} \nabla_{a} K_{b}+\nabla_{b} \nabla^{b} K_{a}-2 k g_{a b} \nabla^{b} \nabla_{c} K^{c}\right) \\
& -\xi_{2}(\kappa) \nabla^{b} y_{\star}^{a}\left(\nabla_{a} K_{b}+\nabla_{b} K_{a}-2 k g_{a b} \nabla_{c} K^{c}\right) \Theta^{\prime} \\
& \left.+\xi_{3}(\kappa) y_{\star}^{a} \Theta^{\prime}\left(\nabla^{b} \nabla_{a} K_{b}+\nabla^{b} \nabla_{b} K_{a}-2 k g_{a b} \nabla^{b} \nabla_{c} K^{c}\right)\right] .
\end{aligned}
$$

Further, the condition for numerical consistency of the generating functional given in (34) together with Eqs. (38) and (40) yields

$$
\begin{aligned}
& \int d^{4} x\left[\left(\frac{d \xi_{1}}{d \kappa}-\frac{\varepsilon}{2} \gamma \sqrt{-g}\right)\left(y_{\star}^{a}+\lambda b^{a}\right)^{2}+\left(\frac{d \xi_{2}}{d \kappa}-\gamma \sqrt{-g}\right) \nabla^{b} y_{\star}^{a}\left(\nabla_{a} y_{b}+\nabla_{b} y_{a}-2 k g_{a b} \nabla_{c} y^{c}\right)\right. \\
& +\left(\frac{d \xi_{3}}{d \kappa}-\gamma \sqrt{-g}\right) K_{\star}^{a}\left(\nabla^{b} \nabla_{a} K_{b}+\nabla_{b} \nabla^{b} K_{a}-2 k g_{a b} \nabla^{b} \nabla_{c} K^{c}\right) \\
& \left.-\left(\xi_{2}-\xi_{3}\right) \nabla^{b} y_{\star}^{a}\left(\nabla_{a} K_{b}+\nabla_{b} K_{a}-2 k g_{a b} \nabla_{c} K^{c}\right) \Theta^{\prime}\right]=0 .
\end{aligned}
$$

By equating the coefficients of various terms present in the above expression from LHS to RHS, we get following linear differential equations:

$$
\frac{d \xi_{1}}{d \kappa}-\gamma \frac{\varepsilon}{2} \sqrt{-g}=0
$$




$$
\begin{aligned}
& \frac{d \xi_{2}}{d \kappa}-\gamma \sqrt{-g}=0 \\
& \frac{d \xi_{3}}{d \kappa}-\gamma \sqrt{-g}=0
\end{aligned}
$$

However, the non-local ( $\Theta^{\prime}$-dependent) terms vanish leading to the following constraints to the parameters:

$$
\xi_{2}-\xi_{3}=0
$$

The solutions of linear differential equations satisfying the initial boundary conditions $\left(\left.\xi_{i}\right|_{\kappa=0}=0, i=\right.$ $1,2,3)$ are

$$
\xi_{1}(\kappa)=\frac{\varepsilon}{2} \sqrt{-g} \kappa, \quad \xi_{2}(\kappa)=\sqrt{-g} \kappa, \quad \xi_{3}(\kappa)=\sqrt{-g} \kappa,
$$

where we have set the arbitrary constant parameter $\gamma=1$. With these solutions, $S_{1}[\phi(x, \kappa), \kappa]$ at $\kappa=1$ (under FFBRST transformation) have the following form:

$$
\begin{aligned}
S_{1}[\phi(x), \varphi(x)]_{\kappa=1} & =\int d^{4} x \sqrt{-g}\left[\frac{\varepsilon}{2}\left(y_{\star}^{a}+\lambda b^{a}\right)^{2}+\nabla^{b} y_{\star}^{a}\left(\nabla_{a} y_{b}+\nabla_{b} y_{a}-2 k g_{a b} \nabla_{c} y^{c}\right)\right. \\
& \left.+K_{\star}^{a}\left(\nabla^{b} \nabla_{a} K_{b}+\nabla_{b} \nabla^{b} K_{a}-2 k g_{a b} \nabla^{b} \nabla_{c} K^{c}\right)\right] .
\end{aligned}
$$

Since $S_{1}[\phi(x), \varphi(x)]_{\kappa=1}$ appears at the exponent of Jacobian (33). Hence, this $S_{1}[\phi(x), \varphi(x)]_{\kappa=1}$ (within functional integral) accumulates to the effective action for perturbative gravity given in (9) leading to an extended action as follows:

$$
\int d^{4} x \mathcal{L}_{T}+S_{1}[\phi(x, 1), \varphi(x, 1), 1]=\int d^{4} x \mathcal{L}_{y k b},
$$

which is exactly the BRST symmetric gaugeon action of perturbative gravity. It means that the Jacobian of measure of functional integral under generalized BRST transformation leads to complete BRST symmetric action of perturbative gravity written in gaugeon fields.

\section{CONCLUSIONS}

We have discussed the gauge invariance and BRST symmetry of perturbative quantum gravity in the Einstein curved spacetime, particularly, in the covariant gauge condition. Further, we have perused the Yokoyama gaugeon formalism for the theory of quantum gravity which extends the effective action by incorporating the quantum (gaugeon) fields. This extended action respects the quantum gauge transformation under which the gauge parameter gets shifted which is claimed as the renormalized gauge parameter by drawing the analogy with ordinary gauge theory. Therefore, such an observation may help in studying the renormalizability of the quantum theory of gravity. Although gaugeon fields discuss the quantum gauge freedom, it does not contribute to physical processes. So we have removed them by employing the Gupta-Bleuler type subsidiary condition. But, unlike the Kugo-Ojima type condition the Gupta-Bleuler condition has certain limitation. Furthermore, this limitations have resolved with the construction of BRST symmetric gaugeon formalism in which we further extend the configuration space by employing corresponding ghost fields too. This extended action possesses an extended BRST symmetry where gaugeon fields form BRST quartet. Within this framework, two Yokoyama's physical subsidiary conditions get replaced by a single Kugo-Ojima type condition. We have demonstrated the quantum gauge transformation for the BRST symmetric gaugeon effective action too which commutes with the extended BRST symmetry. As a result, the physical Hilbert space for perturbative quantum gravity remains unchanged under the quantum gauge transformations.

Further we have generalized the extended BRST symmetry of the perturbative gravity by allowing the parameter to be finite and field-dependent. Doing so, we have found that such transformation changes 
the Jacobian of functional integral non-trivially. However, for a suitable choice of finite field-dependent parameter the Jacobian has led to the gaugeon mode in the perturbative theory of gravity. We have established the results by explicit calculations. This signifies that generalized BRST symmetry with an appropriate parameter will be helpful to describe the theory in gaugeon mode. It will be interesting to generalize the quantum gauge transformation in the same fashion as the generalized BRST transformation which may lead to some new results.

[1] S. Weinberg, "Gravitation and Cosmology", (John Wiley and Sons, New York-1972).

[2] S.W. Hawking and G. F. Ellis, "The Large-Scale Structure Of Space-Time" (Cambridge: Cambridge University Press, 1973).

[3] A. H. Guth, Phys. Rev. D 23, 347 (1981).

[4] A. D. Linde, Phys. Lett. B 108, 389 (1982).

[5] A. Albrecht and P. J. Steinhardt Phys. Rev. Lett. 48, 1220 (1982).

[6] S. Perlmutter, G. Aldering, G. Goldhaber, R. A. Knop, and P. Nugent, et al, Astrophys. J 517, 565 (1999).

[7] A. Einstein, "The Meaning of Relativity, fifth edition (Princeton University Press, 1956).

[8] A. H. Chamseddine, Int. J. Mod. Phys. A 16, 759 (2001).

[9] T. Damour, S. Deser and J. McCarthy, Phys. Rev. D 47, 1541 (1993).

[10] A. H. Chamseddine, Commun. Math. Phys. 218, 283 (2001).

[11] M. Henneaux and C. Teitelboim, Quantization of gauge systems (Princeton, USA: Univ. Press, 1992).

[12] S. D. Joglekar and B. P. Mandal, Phys. Rev. D 51, 1919 (1995).

[13] S. D. Joglekar and B. P. Mandal, Int. J. Mod. Phys. A 17, 1279 (2002).

[14] R. Banerjee and B. P. Mandal, Phys. Lett. B 27, 488 (2000).

[15] S. Upadhyay, S. K. Rai and B. P. Mandal, J. Math. Phys. 52, 022301 (2011).

[16] S. D. Joglekar and A. Misra, Int. J. Mod. Phys. A 15, 1453 (2000).

[17] S. Upadhyay and B. P. Mandal, Mod. Phys. Lett. A 25, 3347 (2010); EPL 93, 31001 (2011); AIP Conf. Proc. 1444, 213 (2012); Eur. Phys. J. C 72, 2065 (2012); Annals of Physics 327, 2885 (2012).

[18] S. Upadhyay, M. K. Dwivedi and B. P. Mandal, Int. J. Mod. Phys. A 28, 1350033 (2013).

[19] M. Faizal, B. P. Mandal and S. Upadhyay, Phys. Lett. B 721, 159 (2013).

[20] B. P. Mandal, S. K. Rai and S. Upadhyay, EPL 92, 21001 (2010).

[21] R. Banerjee, B. Paul and S. Upadhyay, Phys. Rev. D 88, 065019 (2013).

[22] S. Upadhyay, Phys. Lett. B 727, 293 (2013).

[23] R. Banerjee and S. Upadhyay, arXiv:1310.1168 [hep-th].

[24] S. Upadhyay, Annals of Physics 340, 110 (2014).

[25] M. Faizal, Found. Phys. 41, 270 (2011).

[26] S. Upadhyay, Phys. Lett. B 723, 470 (2013).

[27] M. Hayakawa and K Yokoyama, Prog. Theor. Phys. 44, 533 (1970).

[28] K. Yokoyama, Prog. Theor. Phys. 51, 1956 (1974).

[29] K. Yokoyama, Prog. Theor. Phys. 59, 1699 (1978); Prog. Theor. Phys. 60, 1167 (1978); Phys. Lett. B 79,79 (1978).

[30] K. Yokoyama and R. Kubo, Prog. Theor. Phys. 52, 290 (1974).

[31] K. Yokoyama, M. Takeda and M. Monda, Prog. Theor. Phys. 60, 927 (1978).

[32] K. Yokoyama, M. Takeda and M. Monda, Prog. Theor. Phys. 64, 1412 (1980).

[33] K. Izawa, Prog. Theor. Phys. 88, 759 (1992).

[34] M. Koseki, M. Sato and R. Endo, Prog. Theor. Phys. 90, 1111 (1993).

[35] T. Kugo and I. Ojima, Prog. Theor. Phys. Supplement No. 66, 1 (1979).

[36] T. Kugo, I. Ojima, Nucl.Phys. B 144, 234 (1978).

[37] S. Upadhyay, Eur. Phys. J. C 74, 2737 (2014).

[38] S. Upadhyay, EPL 105, 21001 (2014).

[39] M. Koseki, M. Sato and R. Endo, Bull. of Yamagata Univ., Nat. Sci. 14, 15 (1996).

[40] Y. Nakawaki, Prog. Theor. Phys. 98, 5 (1997).

[41] R. Endo and M. Koseki, Prog. Theor. Phys. 103, 3, (2000).

[42] H. Miura and R Endo, Prog. Theor. Phys. 117, 4, (2007).

[43] M. Faizal, Commun. Theor. Phys. 57, 637 (2012).

[44] M. Faizal, Mod. Phys. Lett. A 27, 1250147 (2012).

[45] M. Faizal, A. Higuchi, Phys. Rev. D 78, 067502 (2008). 
[46] T. Kugo and I. Ojima, Phys. Lett. B 73, 459 (1978); Prog. Theor. Phys. 60, 1869 (1978).

[47] A. Higuchi and S. S. Kouris, Class. Quant. Grav. 18, 4317 (2001). 